\title{
Safety of Industrial Applications with Sensitive Mobile Manipulators - Hazards and Related Safety Measures
}

\author{
Andreas Schlotzhauer ${ }^{1}$, Lukas Kaiser ${ }^{1}$ and Mathias Brandstötter ${ }^{1}$
}

\begin{abstract}
The areas of application of robot systems are gradually expanding and mobile manipulation is an important and consistent further development for industrial applications. Although human-robot interaction with these systems becomes easier, the mechatronic design, the integration and safety regarding real applications remain challenging. This paper describes identified dangers and possible hazards of industrial mobile robot systems and sensitive mobile manipulators. Based on a study of advanced sensor technologies and safety concepts, solutions and measures for risk reduction are proposed to counteract these risks. As a key element in mobile robotics, common drive architectures are evaluated with regard to their impact on the general application safety.
\end{abstract}

\section{INTRODUCTION}

With the focus on Industry 4.0 and the associated increasing digitization of the supply chain, there is a high demand for versatile tools in the manufacturing industry [1], [2]. This development includes robot systems that can be used flexibly in such environments. The relatively new field of sensitive mobile manipulation has evolved through major advances in technology and the related development of collaborative manipulators, which fills an aspect of these needs. Such robotic systems have to satisfy a multitude of basic requirements and general conditions, which are examined in this work.

\section{A. Abilities of Mobile Manipulators}

Mobile manipulators, sometimes simply called mobile robots, are the fusion of sensitive manipulators and mobile platforms. Therefore, they combine the two major advantages of both technologies: (i) the capability of working in close proximity to the human, which enables collaboration, and (ii) autonomous relocation and adaptation to a changing environment, which results in novel industrial applications like discussed in [3] and [4].

\section{B. Norms and Standards}

The safety requirements for all types of machinery, within the European Union, is regulated by the so called Machinery Directive [5]. The ISO 12100 [6] is harmonised with the directive and gives general guidance for the safety throughout the life cycle of a machine. One of the main aspects of these documents is the risk-assessment and -reduction of a machine before first operation. The ISO 10218 [7], [8] extends the previously mentioned general standard and also

\footnotetext{
${ }^{1}$ All authors are with JOANNEUM RESEARCH ROBOTICS - Institute for Robotics and Mechatronics - Mechatronic Systems Group, Austria <firstname. lastname>cjoanneum.at
}

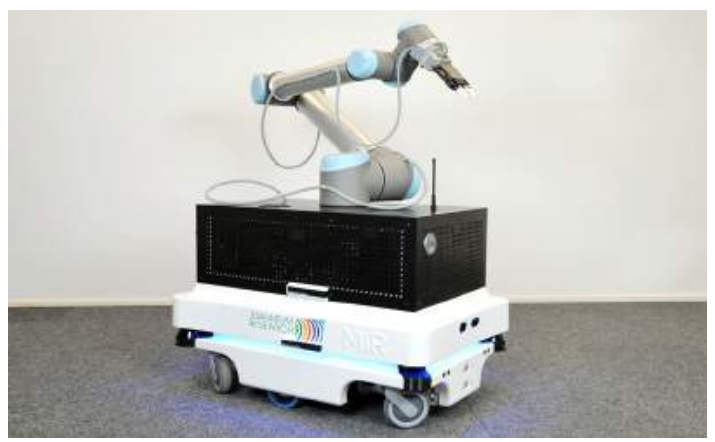

Fig. 1. Mobile manipulator CHIMERA from JOANNEUM RESEARCH

includes more specific safety requirements tailored to the demands of industrial robot applications.

The close vicinity between the robot and the operator in collaborative applications yields new and higher risks. For this reason, the International Organization for Standardization (ISO) released a Technical Specification ISO/TS 15066 [9] addressing the special issues of collaborative robots. For Automated Guided Vehicles (AGVs) on their own two rather old European standards ([10], [11]) exist. These standards are currently revised by the ISO to form the ISO/DIS 36914 [12]. Moreover, at the moment there is only one active standard [13] that directly considers the overall system of a mobile manipulator, but only in the context of personal care and therefore excluding industrial use.

To fill this gap, the sub-committee R15.08, of the American Robotic Industries Association (RIA), is currently developing a new standard for "Mobile Robot Safety". At this time, the developers and integrators of such system are responsible to go beyond the current standards to make their products as safe as possible.

\section{Market Overview}

As mentioned above, the mobility of a mobile manipulator is one of its key advantages over conventional mobile industrial robots. To be able to operate on the shop floor, next to and hand in hand with human workers, the requirements for localization and navigation are high. Although all mobile robots on the market have some kind of these features, it is the quality that sets them apart. Other distinguishing features are, for instance, the maximum loading capacity, runtime, charging time, travel speed, and the quality of 
maps created by integrated SLAM algorithms. In terms of the safety relevant wheel configuration, there are no major differentiations, as most systems use either a differential drive with additional castors or four omnidirectional wheels for increased stability (see section III-A). Examples for the various platform types are, e.g., CHIMERA by JOANNEUM RESEARCH (see Fig. 1) that is based on a differential drive and the KMR iiwa by KUKA as an example for a platform with an omnidirectional drive.

\section{HAZARDS RELATED TO MOBILE MANIPULATION}

There are some common hazards that can occur in every electro-mechanical system, like sharp edges, collision by moving machinery parts, the chance of getting in contact with high voltage or hot/cold surfaces, as well as loud noise, radiation or vibration (for more details see [6]). In the following special hazardous situations are discussed, that can occur only or especially in industrial robotic applications with mobile manipulators.

\section{A. Hazardous Situations}

One source of danger is the movement of a mobile manipulator, more specific the movement of the mobile platform, the attached manipulator or both together. A major risk is the collision (transient and quasi-static contact) with a human, which can only be managed with supplementary measurements.

Another challenge and source of danger is the stability of the whole robot during driving and handling of objects. Especially when the mobile platform and the manipulator are moving simultaneously, the dynamic of the whole robot needs to be taken into account. When the movement of the robot is limited (e.g., the robot fell over, the remaining stored energy is not sufficient, the drive is damaged), the robot should still be manually movable, as it could block an emergency escape route or be a barrier for other vehicles or humans. On the other hand the robot should not move unintentionally while being on an uneven surface or doing a precise task with its end-effector, as this could also lead to further accidents.

Even when the risk of a collision is reduced with sensors, there might still be a chance for hazardous situations. This could happen, e.g., when the robot converges to a docking station, its view is blocked by obstacles and objects are not visible from the robots point of view. This is also relevant for objects carried by the robot.

Dynamic changes in the environment and unknown objects in that environment could lead to situations, that where not predicted during integration and therefore, are not covered in the previously performed risk assessment. The interaction with dangerous objects/tools and the presence in unsuitable areas can hardly be completely excluded.

A communication between the robot system and humans tailored to the application should be considered to avoid confusion and misunderstanding and therefore to decrease the probability of the occurrence of a hazardous situation.
More specifically, this means that one cannot assume that only qualified persons will interact with the robot (e.g., visitor groups that are guided through a production hall).

\section{B. Possible Injuries}

There is a wide scope of injuries that could occur due to the described hazardous situations. Special attention should be given to the high possibility of collisions between a robot and a human, as this is a unique property to collaborative robot applications. [14] studies possible soft tissue injuries and in [9] thresholds for the human experience of pain are given. For possible contact situations of an application, the compliance with these thresholds can be verified, to ensure the prevention of any injury [15].

\section{DESIGN CONCEPTS}

In order to design a robot system for industrial use, the safety aspects must be taken into account from the very beginning. Measures to reduce the risks, identified by a risk assessment [6], are grouped and prioritized into

1) Inherently safe design,

2) Complementary measures and

3) Organisational measures.

In the following, major concepts are presented to design and safeguard an industrial mobile application in different aspects.

\section{A. Drive of Mobile Robots}

An important element of a mobile platform or a mobile manipulator and therefore for the whole system is the locomotion mechanism, typically a drive, of the robot. If we restrict ourselves to wheeled mobile robots, then there are 4 basic types of wheels: standard wheels, castor wheels, Swedish wheels and spherical wheels. Each having different sliding and rolling constraint and affecting the maneuverability and controllability of the drive differently [16]. It is desirable, that the mobile manipulator is static stable and does not tilt during driving. A hyperstatic wheel geometry could lead to loose control on uneven floor. In general, omnidirectional drives in contrast to differential drives allow to react more flexible to dynamic changes in the planned path and are more suitable for narrow workspaces but could lead to undesired movements without active control (e.g. on an uneven floor). In table I common drives, presented in [16], are evaluated concerning their implication to safety, when used in a mobile industrial application. Also particular realizations can have different properties, and therefore, the general tendency is described.

\section{B. Design of the Robot}

Concerning the mechanical design of the whole robot and end-effector, safety should be considered from the early beginning. The identified main mechanical design concepts related to safety are

- Lightweight design,

- Rounded edges,

- Compliant covering, 
- Maximizing potential collision surfaces,

- Excluding bruise and shear of body parts,

- Limited workspace.

Also the physical interaction between the robot and a human is neither necessary nor scheduled or even prevented by supplementary measurements. It has to be noted, that a contact could still occur by intentional missus or due to a failure. In terms of safety (and efficiency) the whole robot should be as light as possible, especially all moving parts of the manipulator. For a good stability the center of gravity should be near the ground. Rounded edges and a soft covering can not only decrease the collision force and pressure, but also give a more comfortable feeling while touching the robot, which again could lead to higher acceptance by the operators. The bruise and shear of human limbs should be impossible anyway, not at least for the manual repair and maintenance of the robot. Rounded surfaces can also prevent

TABLE I

SAFETY OF COMMON DRIVES

(a)

\begin{tabular}{c|c}
$\square$ & Powered standard wheel \\
\hline$\bigcirc$ & Passive spherical wheel \\
\hline$\square$ & Passive standard wheel \\
\hline$\square \square$ & Powered Swedish wheel
\end{tabular}

that objects can be placed on the robot and fall down during driving. The area where the robot can move should not be larger than necessary and also the manipulator workspace can be limited if possible.

\section{Gripping}

Especially the end-effector of a mobile manipulator, often a gripper, should be designed following the above listed principles, since in many cases the end-effector is the only physical interface with the environment (except the wheels) and the human. In mobile manipulation form-fit gripping should be preferred over force-fit gripping, as a gripped object cannot be lost after power loss, when it is slippery or even with a dynamic movement. By monitoring the gripping force and displacement of the gripper fingers, the compliance of the grasp object can be determined and the presence of human limbs can be detected. If gripping tasks or the handling of tools require fine mechanics or sharp edges, the covering or flexible suspension of the whole end-effector can be a solution [17]. In some applications flexible gripper fingers or a suctions cup can avoid sharp edged, but they might lack in precision and payload.

\section{AVAILABLE SAFETY TECHNOLOGY}

As mentioned in the previous chapter, one possibility to reduce a risk is to put complementary measures into place. Historically, that is understood to putting the robot behind a ridged or light fence. With mobile manipulators this is usually not possible, and hence, the safety relies heavily on several modern sensor technologies which are presented in this section.

\section{A. Localisation, Planning and Navigation}

One key-aspect for safety in mobile robots is their capability of recognising their surroundings and acting to that accordingly. By constantly mapping its surrounding and localizing its position (simultaneous localization and mapping (SLAM)) the mobile robot is able to navigate safely in unstructured environments without collisions. To improve the localization and thereby also the safety, artificial features (bar codes, QR-tags, magnets etc.) can be used, although they might be covered by obstacles. On the other hand, natural landmarks are more challenging to detect, but with the advantage of lower risk of manipulation, damaging or covering.

\section{B. Sensors}

Regarding the risk, the sensor addresses, a suitable sensor technology has to be chosen. Different sensor types cover different aspects of the real world and can be distinguished by their robustness against external factors. In the following common sensor types and their impact on application-safety are presented. 
1) Odometry: The major advantage of common odometry sensors like rotary encoders or accelerometers, is the high robustness, due to the basic underlying principles. Because the measurement is relative to the last information (except absolute rotary encoders), the error accumulate and the reliability of the sensor information decreases over time, which can be stabilized with extra reference points (global reference). When the position of the robot is derived from wheel rotation, slipping distorts the position accuracy until the next absolute reference. The computational power, needed for evaluation, is relatively low.

2) Tactile Sensing: A mechanical switch is very robust, although the derived information is simple. With higher complexity more sophisticated information can be captured. Besides the usage of tactile sensors in external input devices, artificial robot skins enable tactile sensing to standard robots, with the help of pressure sensitive air cushion or distributed and flexible force sensing elements on the robot surface. In that way the contact with humans can be detected and the avoidance of injuries is possible by appropriate reactions. The contact with the environment can also be perceived by force-torque measurements in the robot joints or base. This method might be difficult or even useless when a stationary robot is mounted on a mobile platform, without concerning and modeling the dynamic of the whole mobile manipulator.

3) Distance Sensing: There are several sensors available based on Time of Flight (TOF) principle for measuring distances like SONAR, LIDAR and RADAR. Known issues with such systems are crosstalk, multi-reflections, absorption/permeability or insufficient reflection. Environmental conditions, e.g. sunlight or glass walls, can also decrease the performance.With a suitable arrangement of capacitive sensors, the orientation and distance to obstacles or people in the immediate vicinity can be calculated [18].

\section{Sensor Fusion}

The basic idea behind sensor fusion related to safety is to increase the coverage or integrity of the extracted information from different sensors by combining several sources of data. The combination of different types of sensors based on different operating principles decreases the chance of malfunction related to a common cause. This is also crucial for the redundancy requirements of safe interaction with the environment. In case of a mismatch between two channels the trustworthiness is not given any more for both signals and therefore the derived information as well. By cross checking more than two channels the failure of one specific signal can be recognized with high probability. The difference between channels can also be caused by the limited capabilities of different operating principles, e.g., detecting a pane of glass with an optical sensor versus an ultrasonic sensor. This reduces the trustworthiness of the consolidated sensor data but increases the scope of perceivable information. It is not trivial to distinguish between these two situations, however it can be achieved by pairing two similar sensors for each operating principle.

\section{Safety of dynamic workflow}

Due to undetermined dynamic changes in flexible mobile robotic applications, not every possible situation can be analysed regarding its risk beforehand. Therefore some kind of dynamic risk analysis during runtime would be beneficial. To realize this approach some kind of intelligent system is necessary to be aware of the situation and assess the same. Image classification/object recognition is widely used to achieve this goal. Neuronal networks are able to find dependencies within vast datasets (e.g., image collection) which can be used to evaluate new situations. This results in high level information that can not be derived from any other sensor with the drawback of not being replicable and therefore also not predictable, which is problematic for safety related functionalities. Potential fields with risk sources can be used to react and re-plan actions [19].

\section{E. Multistage Safety Concept}

As different types of sensors have different levels of reliability, a multistage concept can be used to increase productivity without sacrificing safety. Such systems could switch automatically between different safe modes depending on sensor input (e.g., distances) and the state of the available safety features (e.g., trustworthiness, failures), keeping the productivity as high as possible. For example, the use of an AI based vision system increases the predictability of the movement of humans, if the feature can not be trusted any more or fails, the system can then reduce the speed by relying on, e.g., the still working LIDAR scanners without beeing forced to stop the system.

\section{CONCLUSIONS}

The dissemination of flexible mobile application comes with chances and risks. While mobile manipulation is highly developed in research labs, industrial application remain tough, due to the lack of reference standards and experiencebased knowledge. To face hazards in dynamic environments, a solid design that increases inherent safety is a fundamental requirement for a safe application. Also a suitable mechanical design is not enough. Instead, only advanced sensor technology or even AI-based methods can achieve a high level of safety, but in contrast they are error-prone and difficult to maintain. Redundancy and the combination of different technologies is crucial to overcome this problems. A good safety concept should not hinder the advanced possibilities of mobile manipulation, whereby operational safety should be in the foreground. To achieve this, knowledge of hazards and countermeasures must be transferred from the laboratory to the integrators and operators.

\section{ACKNOWLEDGMENT}

The results incorporated in this paper were gained within the scope of the project "HRC-Safety for employees" commissioned by the Allgemeine Unfallversicherungsanstalt (AUVA). 


\section{REFERENCES}

[1] H. Hirsch-Kreinsen, "Digitization of industrial work: development paths and prospects," Journal for Labour Market Research, vol. 49, pp. $1-14,2016$.

[2] D. Wurhofer, T. Meneweger, V. Fuchsberger, and M. Tscheligi, "Reflections on operators and maintenance engineers experiences of smart factories," in Proceedings of the 2018 ACM Conference on Supporting Groupwork, 2018, pp. 284-296.

[3] K. Zhou, G. Ebenhofer, C. Eitzinger, U. Zimmermann, C. Walter, J. Saenz, L. P. Castao, M. A. F. Hernndez, and J. N. Oriol, "Mobile manipulator is coming to aerospace manufacturing industry," in 2014 IEEE International Symposium on Robotic and Sensors Environments (ROSE) Proceedings, Timisoara, Romania, Oct. 2014, pp. 94-99.

[4] FLEXIFF consortium. (2018, Mar.) Flexiff - flexible intralogistics for future factories. [Online]. Available: http://www.flexiff.at/

[5] "Directive 2006/42/EC of the European Parliament and of the Council of 17 May 2006 on machinery, and amending Directive 95/16/EC (recast)," The European Parliament and the Council of The European Union, Brussels, Belgium, 2006.

[6] "ISO 12100:2010-11 Safety of machinery - General principles for design - Risk assessment and risk reduction," International Organization for Standardization (ISO), Geneva, Switzerland, 2013.

[7] "ISO 10218-1:2011-07 Robots and robotic devices - Safety requirements for industrial robots - Part 1: Robots," International Organization for Standardization (ISO), Geneva, Switzerland, 2012.

[8] "ISO 10218-2:2011-07 Robots and robotic devices - Safety requirements for industrial robots - Part 2: Robot systems and integration," International Organization for Standardization (ISO), Geneva, Switzerland, 2012

[9] "ISO/TS 15066:2016-02 Robots and robotic devices - Collaborative robots," International Organization for Standardization (ISO), Geneva, Switzerland, 2016.

[10] "EN 1525:1997-09 Safety of industrial trucks - Driverless trucks and their systems," European Committee for Standardization (CEN), Brussels, Belgium, 1997.

[11] "EN 1526:1997-09 Safety of industrial trucks - Additional requirements for automated functions on trucks," European Committee for Standardization (CEN), Brussels, Belgium, 1997.

[12] "ISO/DIS 3691-4 Industrial trucks - Safety requirements and verification - Part 4: Driverless industrial trucks and their systems," International Organization for Standardization (ISO), Geneva, Switzerland, 2018.

[13] "ISO 13482:2014-02 Robots and robotic devices - Safety requirements for personal care robots," International Organization for Standardization (ISO), Geneva, Switzerland, 2014.

[14] S. Haddadin, A. Albu-Schäffer, and G. Hirzinger, "Soft-tissue injury in robotics," in Proceedings of the 2010 IEEE International Conference on Robotics and Automation, 2010.

[15] "Kollaborierende Robotersysteme - Planung von Anlagen mit der Funktion Leistungs- und Kraftbegrenzung FB HM-080," Deutsche Gesetzliche Unfallversicherung (DGUV), Berlin, Germany, 2017.

[16] R. Siegwart, I. R. Nourbakhsh, and D. Scaramuzza, Introduction to autonomous mobile robots. MIT press, 2011.

[17] R. Weitschat, J. Vogel, S. Lantermann, and H. Höppner, "Endeffector airbags to accelerate human-robot collaboration," in IEEE International Conference on Robotics and Automation (ICRA). IEEE, 2017, pp. 2279-2284.

[18] M. Brandstötter, S. Mühlbacher-Karrer, D. Schett, and H. Zangl, "Virtual compliance control of a kinematically redundant serial manipulator with 9 dof," in Advances in Robot Design and Intelligent Control. RAAD 2016, 2016, pp. 38-46.

[19] B. Lacevic and P. Rocco, "Kinetostatic danger field - a novel safety assessment for human-robot interaction," in International Conference on Intelligent Robots and Systems (IROS), 2010 IEEE/RSJ, Taipei, Taiwan, 2010, pp. 2169-2174. 
